\title{
On monotone contraction mappings in modular function spaces
}

\author{
Monther R Alfuraidan ${ }^{1}$, Mostafa Bachar ${ }^{2 *}$ and Mohamed A Khamsi ${ }^{3}$
}

"Correspondence:

mbachar@ksu.edu.sa

${ }^{2}$ Department of Mathematics, College of Sciences, King Saud University, Riyadh, Saudi Arabia Full list of author information is available at the end of the article

\begin{abstract}
We prove the existence of fixed points of monotone-contraction mappings in modular function spaces. This is the modular version of the Ran and Reurings fixed point theorem. We also discus the extension of these results to the case of pointwise monotone-contraction mappings in modular function spaces.
\end{abstract}

MSC: Primary 47H09; secondary 47H10

Keywords: fixed point; modular function space; monotone mappings; pointwise contraction

\section{Introduction}

The purpose of this paper is to give an outline of a fixed point theory for monotonecontraction mappings defined on some subsets of modular function spaces which are natural generalizations of both function and sequence variants of many important spaces like the Lebesgue, Orlicz, Musielak-Orlicz, Lorentz, Orlicz-Lorentz, Calderon-Lozanovskii spaces, and many others [1]. Recently, the authors in [2] presented a series of fixed point results for pointwise contractions and asymptotic pointwise contractions acting in modular functions spaces. The current paper operates within the same framework.

The importance for applications of mappings defined within modular function spaces consists in the richness of the structure of modular function spaces, which, besides being Banach spaces, are equipped with modular equivalents of norm or metric notions and also are equipped with almost everywhere convergence and convergence in sub-measure. In many cases, particularly in applications to integral operators, approximation and fixed point results, modular type conditions are much more natural as modular type assumptions can be more easily verified than their metric or norm counterparts; see for example [3]. From this perspective, the fixed point theory in modular function spaces should be considered as complementary to the fixed point theory in Banach linear spaces and in metric spaces.

The theory of contractions and nonexpansive mappings defined on convex subsets of Banach spaces has been well developed since the 1960s, see for example [4-7], and generalized to other metric spaces [8-10], and modular function spaces [3,11]. The corresponding fixed point results were then extended to larger classes of mappings like asymptotic mappings [12,13], pointwise contractions [14], and asymptotic pointwise contractions and nonexpansive mappings [2, 15-17].

( 2015 Alfuraidan et al: licensee Springer. This is an Open Access article distributed under the terms of the Creative Commons Attribution License (http://creativecommons.org/licenses/by/4.0), which permits unrestricted use, distribution, and reproduction in any medium, provided the original work is properly credited. 
In recent years, a version of the Banach contraction principle [18] was given in partially ordered metric spaces $[19,20]$ and in metric spaces with a graph [21]. In this work, we discuss some of these extensions to modular function spaces.

\section{Preliminaries}

Let $\Omega$ be a nonempty set and $\Sigma$ be a nontrivial $\sigma$-algebra of subsets of $\Omega$. Let $\mathcal{P}$ be a $\delta$-ring of subsets of $\Omega$, such that $E \cap A \in \mathcal{P}$ for any $E \in \mathcal{P}$ and $A \in \Sigma$. Let us assume that there exists an increasing sequence of sets $K_{n} \in \mathcal{P}$ such that $\Omega=\bigcup K_{n}$. By $\mathcal{E}$ we denote the linear space of all simple functions with supports from $\mathcal{P}$. By $\mathcal{M}_{\infty}$ we will denote the space of all extended measurable functions, i.e. all functions $f: \Omega \rightarrow[-\infty, \infty]$ such that there exist a sequence $\left\{g_{n}\right\} \subset \mathcal{E},\left|g_{n}\right| \leq|f|$ and $g_{n}(\omega) \rightarrow f(\omega)$ for all $\omega \in \Omega$. By $1_{A}$ we denote the characteristic function of the set $A$.

Definition 2.1 Let $\rho: \mathcal{M}_{\infty} \rightarrow[0, \infty]$ be a nontrivial even function. We say that the regular function $\rho$ is pseudomodular if:

(i) $\rho(0)=0$;

(ii) $\rho$ is monotone, i.e. $|f(\omega)| \leq|g(\omega)|$ for all $\omega \in \Omega$ implies $\rho(f) \leq \rho(g)$, where $f, g \in \mathcal{M}_{\infty}$

(iii) $\rho$ is orthogonally additive, i.e. $\rho\left(f 1_{A \cup B}\right)=\rho\left(f 1_{A}\right)+\rho\left(f 1_{B}\right)$, for any $A, B \in \Sigma$ such that $A \cap B=\emptyset, f \in \mathcal{M}$;

(iv) $\rho$ has the Fatou property, i.e. $\left|f_{n}(\omega)\right| \uparrow|f(\omega)|$ for all $\omega \in \Omega$ implies $\rho\left(f_{n}\right) \uparrow \rho(f)$, where $f \in \mathcal{M}_{\infty}$;

(v) $\rho$ is order continuous in $\mathcal{E}$, i.e. $g_{n} \in \mathcal{E}$ and $\left|g_{n}(\omega)\right| \downarrow 0$ implies $\rho\left(g_{n}\right) \downarrow 0$.

Similar to the case of measure spaces, we say that a set $A \in \Sigma$ is $\rho$-null if $\rho\left(g 1_{A}\right)=0$ for every $g \in \mathcal{E}$. We say that a property holds $\rho$-almost everywhere if the exceptional set is $\rho$-null. As usual, we identify any pair of measurable sets whose symmetric difference is $\rho$-null as well as any pair of measurable functions differing only on a $\rho$-null set. With this in mind we define

$$
\mathcal{M}(\Omega, \Sigma, \mathcal{P}, \rho)=\left\{f \in \mathcal{M}_{\infty} ;|f(\omega)|<\infty \rho \text {-a.e. }\right\}
$$

where each $f \in \mathcal{M}(\Omega, \Sigma, \mathcal{P}, \rho)$ is actually an equivalence class of functions equal $\rho$-a.e. rather than an individual function. Where no confusion exists we will write $\mathcal{M}$ instead of $\mathcal{M}(\Omega, \Sigma, \mathcal{P}, \rho)$.

Definition 2.2 Let $\rho$ be a regular function pseudomodular.

(1) We say that $\rho$ is a regular function semimodular if $\rho(\alpha f)=0$ for every $\alpha>0$ implies $f=0 \rho$-a.e.;

(2) we say that $\rho$ is a regular function modular if $\rho(f)=0$ implies $f=0 \rho$-a.e.

The class of all nonzero regular function modulars defined on $\Omega$ will be denoted by $\Re$.

Let us denote $\rho(f, E)=\rho\left(f 1_{E}\right)$ for $f \in \mathcal{M}, E \in \Sigma$. It is easy to prove that $\rho(f, E)$ is a function pseudomodular in the sense of Definition 2.1.1 in [1]. More precisely, it is a function pseudomodular with the Fatou property. Therefore, we can use all results of the standard theory of modular function spaces as per the framework defined by Kozlowski in [1, 22, 23]. 
Definition $2.3[1,22,23]$ Let $\rho$ be a function modular.

(a) A modular function space is the vector space $L_{\rho}(\Omega, \Sigma)$, or briefly $L_{\rho}$, defined by

$$
L_{\rho}=\{f \in \mathcal{M} ; \rho(\lambda f) \rightarrow 0 \text { as } \lambda \rightarrow 0\} .
$$

(b) The following formula defines a norm in $L_{\rho}$ (frequently called the Luxemburg norm):

$$
\|f\|_{\rho}=\inf \{\alpha>0 ; \rho(f / \alpha) \leq 1\} .
$$

In the following theorem we recall some of the properties of modular spaces.

Theorem 2.1 [1,22, 23] Let $\rho \in \Re$.

(1) $\left(L_{\rho},\|\cdot\|_{\rho}\right)$ is complete and the norm $\|\cdot\|_{\rho}$ is monotone w.r.t. the natural order in $\mathcal{M}$.

(2) $\left\|f_{n}\right\|_{\rho} \rightarrow 0$ if and only if $\rho\left(\alpha f_{n}\right) \rightarrow 0$ for every $\alpha>0$.

(3) If $\rho\left(\alpha f_{n}\right) \rightarrow 0$ for an $\alpha>0$ then there exists a subsequence $\left\{g_{n}\right\}$ of $\left\{f_{n}\right\}$ such that $g_{n} \rightarrow 0 \rho$-a.e.

(4) $\rho(f) \leq \liminf \rho\left(f_{n}\right)$ whenever $f_{n} \rightarrow f \rho$-a.e. (Note: this property is equivalent to the Fatou property.)

The following definition plays an important role in the theory of modular function spaces [1].

Definition 2.4 Let $\rho \in \Re$. We say that $\rho$ has the $\Delta_{2}$-property if $\sup _{n} \rho\left(2 f_{n}, D_{k}\right) \rightarrow 0$, whenever $\left\{D_{k}\right\} \in \Sigma$ is decreasing, $\bigcap_{k \geq 1} D_{k}=\emptyset$, and $\lim _{k \rightarrow+\infty} \sup _{n} \rho\left(f_{n}, D_{k}\right)=0$.

Theorem 2.2 Let $\rho \in \Re$. The following conditions are equivalent:

(a) $\rho$ has $\Delta_{2}$,

(b) if $\rho\left(f_{n}\right) \rightarrow 0$ then $\rho\left(2 f_{n}\right) \rightarrow 0$,

(c) if $\rho\left(\alpha f_{n}\right) \rightarrow 0$ for an $\alpha>0$ then $\left\|f_{n}\right\|_{\rho} \rightarrow 0$, i.e. the modular convergence is equivalent to the norm convergence.

We will also use another type of convergence which is situated between norm and modular convergence. It is defined, among other important terms, in the following.

Definition 2.5 Let $\rho \in \Re$.

(a) We say that $\left\{f_{n}\right\}$ is $\rho$-convergent to $f$ and write $f_{n} \rightarrow 0(\rho)$ if and only if $\rho\left(f_{n}-f\right) \rightarrow 0$.

(b) A sequence $\left\{f_{n}\right\}$ where $f_{n} \in L_{\rho}$ is called $\rho$-Cauchy if $\rho\left(f_{n}-f_{m}\right) \rightarrow 0$ as $n, m \rightarrow \infty$.

(c) A set $B \subset L_{\rho}$ is called $\rho$-closed if for any sequence of $f_{n} \in B$, the convergence $f_{n} \rightarrow f(\rho)$ implies that $f$ belongs to $B$.

(d) A set $B \subset L_{\rho}$ is called $\rho$-bounded if $\sup \{\rho(f-g)$; $f \in B, g \in B\}<\infty$.

(e) A set $B \subset L_{\rho}$ is called strongly $\rho$-bounded if there exists $\beta>1$ such that $\sup \{\rho(\beta(f-g)) ; f \in B, g \in B\}<\infty$.

(f) A set $C \subset L_{\rho}$ is called $\rho$-a.e. closed if for any $\left\{f_{n}\right\}$ in $C$ which $\rho$-a.e. converges to some $f$, then we must have $f \in C$.

(g) A set $C \subset L_{\rho}$ is called $\rho$-a.e. compact if for any $\left\{f_{n}\right\}$ in $C$, there exists a subsequence $\left\{f_{n_{k}}\right\}$ which $\rho$-a.e. converges to some $f \in C$. 
Let us note that $\rho$-convergence does not necessarily imply $\rho$-Cauchy condition. Also, $f_{n} \rightarrow f$ does not imply in general $\lambda f_{n} \rightarrow \lambda f, \lambda>1$. Using Theorem 2.1 it is not difficult to prove the following.

Proposition 2.1 Let $\rho \in \Re$.

(i) $L_{\rho}$ is $\rho$-complete.

(ii) $L_{\rho}$ is a lattice, i.e., for any $f, g \in L_{\rho}$, we have $\max \{f, g\} \in L_{\rho}$ and $\min \{f, g\} \in L_{\rho}$.

(iii) $\rho$-balls $B_{\rho}(x, r)=\left\{y \in L_{\rho} ; \rho(x-y) \leq r\right\}$ are $\rho$-closed and $\rho$-a.e. closed.

Using the property (3) of Theorem 2.1, we get the following result.

Theorem 2.3 Let $\rho \in \Re$. Let $\left\{f_{n}\right\}$ be a $\rho$-Cauchy sequence in $L_{\rho}$. Assume that $\left\{f_{n}\right\}$ is monotone increasing, i.e., $f_{n} \leq f_{n+1} \rho$-a.e. (resp. decreasing, i.e., $f_{n+1} \leq f_{n} \rho$-a.e.), for any $n \geq 1$. Then there exists $f \in L_{\rho}$ such that $\rho\left(f_{n}-f\right) \rightarrow 0$ and $f_{n} \leq f \rho$-a.e. (resp. $f \leq f_{n} \rho$-a.e.), for any $n \geq 1$.

Let us finish this section with the modular definitions of some special mappings, which will be studied throughout. The definitions are straightforward generalizations of their norm and metric equivalents [13, 15-17].

Definition 2.6 Let $\rho \in \Re$ and let $C \subset L_{\rho}$ be nonempty and $\rho$-closed. A mapping $T: C \rightarrow$ $C$ is said to be monotone increasing (resp. decreasing) if $T(f) \leq T(g) \rho$-a.e. (resp. $T(g) \leq$ $T(f) \rho$-a.e.) whenever $f \leq g \rho$-a.e., for any $f, g \in C$. $T$ is said to be monotone whenever $T$ is either monotone increasing or decreasing. Moreover, $T$ is called

(i) a monotone contraction if $T$ is monotone and there exists $k<1$ such that

$$
\rho(T(f)-T(g)) \leq k \rho(f-g)
$$

whenever $f, g \in C$ and $f \leq g$-a.e.;

(ii) a pointwise monotone contraction if $T$ is monotone and for any $f \in C$ there exists $k_{f}<1$ such that

$$
\rho(T(f)-T(g)) \leq k_{f} \rho(f-g)
$$

whenever $g \in C$ such that $f \leq g$ or $g \leq f \rho$-a.e.;

(iii) an asymptotic pointwise monotone contraction if $T$ is monotone and for any $f \in C$ and $n \geq 1$, there exists a constant $k_{f}^{n}$ such that

$$
\rho\left(T^{n}(f)-T^{n}(g)\right) \leq k_{f}^{n} \rho(f-g)
$$

with $k_{f}=\lim \sup _{n \rightarrow \infty} k_{f}^{n}<1$, whenever $g \in C$ such that $f \leq g$ or $g \leq f \rho$-a.e.

We shall call a point $f \in C$ a fixed point of $T$ if and only if $T(f)=f$.

Let us give an example which will illustrate the role of the above defined notions. This example is a typical application of the methods of modular function spaces to the theory of nonlinear integral and differential equations. 
Example 2.1 Let us consider a pharmacokinetics sub-model of ascorbic acid (in short AA) absorption in the intestines in healthy subjects developed in [24]; such a model can be written as

$$
\begin{aligned}
& \frac{d A_{\text {gut }}(t)}{d t}=-\frac{\gamma A_{\text {gut }}(t)}{\beta+A_{\text {gut }}(t)}-K_{\text {gut }} A_{\text {gut }}(t), \quad t \neq \tau_{n}, \\
& A_{\text {gut }}\left(\tau_{n}^{+}\right)=A_{\text {gut }}\left(\tau_{n}\right)+D_{A A_{n}} .
\end{aligned}
$$

Here $n \geq 1$ and $A_{\text {gut }}$ represent the amount of AA in the intestines (in $\mu \mathrm{mol}$ ) and the initial condition $A_{\text {gut }}(0)=D$ (in $\mu \mathrm{mol}$ ) is given or estimated by a physician at given steady state, and $0=\tau_{0}<\tau_{1}<\tau_{2}<\cdots<\tau_{n}<\tau_{n+1}<\cdots$, where $\tau_{n}$ represent the time episode of oral intake, $A_{\text {gut }}\left(\tau_{n}^{+}\right)$represents the right limit of $A_{\text {gut }}(t)$ at the point $\tau_{n}$. For practical reasons and without loss of any generality, we will assume that the given dose of oral intake $D_{A A_{n}}$ is $\omega$ periodic and equal to the constant $D$ over $[0,+\infty)$. The description of the model parameters $\gamma, \beta$, and $K_{\text {gut }}$ with their units are given in [24]. Moreover, we find that the solution of (2.2)-(2.3) exists for $t \geq 0$, and it is positive and bounded from above. In fact (2.2)-(2.3) with given initial conditions have a unique solution for all $t \geq 0$. Now let $A_{\text {gut }}(t)$ be a solution of (2.2). If there exists a $t_{0}=\inf \left\{t>0, t \neq \tau_{n}: A_{\text {gut }}(t)<0\right\}$ such that $A_{\text {gut }}\left(t_{0}\right)=0$, then we will have $\left.\frac{d A_{\text {gut }}(t)}{d t}\right|_{t_{0}}<0$ and also from (2.2), we get

$$
0>\left.\frac{d A_{\text {gut }}(t)}{d t}\right|_{t_{0}}=-\frac{\gamma A_{\text {gut }}(t)}{\beta+A_{\text {gut }}(t)}-K_{\text {gut }} A_{\text {gut }}\left(t_{0}\right)=0,
$$

which is a contradiction. Therefore we have $A_{\text {gut }}(t) \geq 0$, for all $t \neq \tau_{n}$, and by using the impulsive equation (2.3) we get $A_{\text {gut }}(t) \geq 0$, for all $t>0$.

Now we will show the boundedness of $A_{\text {gut }}(t)$, for all $t>0$. We have from (2.2)

$$
\frac{d A_{\text {gut }}(t)}{d t} \leq-K_{\text {gut }} A_{\text {gut }}(t), \quad t \neq \tau_{n}, \quad A\left(\tau_{n}^{+}\right)=A_{\text {gut }}\left(\tau_{n}\right)+D_{A A_{n}},
$$

for any $n \geq 1$. Then we have by successive methods

$$
\begin{aligned}
& A_{\text {gut }}(t) \leq\left(\sum_{i=1}^{k} D_{A A_{i-1}} e^{-K_{\text {gut }}\left(h_{i}+h_{i+1}+\cdots+h_{k}\right)}+D_{A A_{k}}\right) e^{-K_{\text {gut }}\left(t-\tau_{k-1}\right)}, \quad \tau_{k-1} \leq t<\tau_{k}, \\
& A_{\text {gut }}(t) \leq D_{A A_{0}}, \quad 0 \leq t<\tau_{1},
\end{aligned}
$$

where $k \geq 2, h_{i}=\tau_{i}-\tau_{i-1}>0, \forall i=1,2, \ldots$, and as we have $D_{A A_{i}}$ is a finite number, then $A_{\text {gut }}(t)$ is bounded from above. It is easy to check that the solution of (2.2)-(2.3) for all $t>\tau_{n}, n \geq 1$, can be written as

$$
\begin{aligned}
A_{\text {gut }}(t) & =\sum_{0 \leq \tau_{n}<t} D_{A A_{n}} e^{-K_{\text {gut }}\left(t-\tau_{n}\right)}-\int_{0}^{t} \frac{\gamma A_{\text {gut }}(s)}{\beta+A_{\text {gut }}(s)} e^{-K_{\text {gut }}(t-s)} d s \\
& =D \sum_{0 \leq \tau_{n}<t} e^{-K_{\text {gut }}\left(t-\tau_{n}\right)}-\int_{0}^{t} \frac{\gamma A_{\text {gut }}(s)}{\beta+A_{\text {gut }}(s)} e^{-K_{\text {gut }}(t-s)} d s .
\end{aligned}
$$

In order to find an $\omega$-periodic solution, where the dose of the treatment will be fixed and equal to $D$, the amount of $A A$ in the intestines needs to satisfy $A_{\text {gut }}(t)=A_{\text {gut }}(t+\omega)$, for all $t \geq 0$. 
Note that by evaluating the periodic condition $A_{\text {gut }}(t)=A_{\text {gut }}(t+\omega)$, we conclude that $A_{\text {gut }}(t)$ is an $\omega$-periodic solution of (2.2)-(2.3) if and only if $A_{\text {gut }}$ is a fixed point of the following operator:

$$
\begin{aligned}
T\left(A_{\text {gut }}\right)(t) & =D \sum_{t \leq \tau_{n}<t+\omega} G\left(t, \tau_{n}\right)-\int_{t}^{t+\omega} G(t, s) \frac{\gamma A_{\text {gut }}(s)}{\beta+A_{\text {gut }}(s)} d s \\
& =D \sum_{t \leq \tau_{n}<t+\omega} G\left(t, \tau_{n}\right)-\int_{t}^{t+\omega} G(t, s) f\left(A_{\text {gut }}(s)\right) d s
\end{aligned}
$$

where $G(t, s)=\frac{e^{-K_{\text {gut }}(t+\omega-s)}}{1-e^{-K_{\text {gut }} \omega}}$ and $f(x)=\frac{\gamma x}{\beta+x}$. Let us consider the following function modular:

$$
\rho(g)=\int_{0}^{\omega}\left\{\int_{t}^{t+\omega} G(t, s)|g(s)| d s\right\} d t
$$

Using the mean-value theorem, we get

$$
\begin{aligned}
0 & \leq-\left(T\left(A_{\text {gut }}\right)(t)-T\left(\widetilde{A}_{\text {gut }}\right)(t)\right)=\int_{t}^{t+\omega} G(t, s)\left(f\left(A_{\text {gut }}(s)\right)-f\left(\widetilde{A}_{\text {gut }}(s)\right)\right) d s \\
& \leq \frac{\gamma}{\beta} \int_{t}^{t+\omega} G(t, s)\left(A_{\text {gut }}(s)-\widetilde{A}_{\text {gut }}(s)\right) d s
\end{aligned}
$$

whenever $\widetilde{A}_{\text {gut }} \leq A_{\text {gut }}$. Hence $T\left(A_{\text {gut }}\right) \leq T\left(\widetilde{A}_{\text {gut }}\right)$ and

$$
\begin{aligned}
\rho\left(T\left(A_{\text {gut }}\right)-T\left(\widetilde{A}_{\text {gut }}\right)\right) & =\int_{0}^{\omega} \int_{t}^{t+\omega} G(t, s)\left(T\left(\widetilde{A}_{\text {gut }}\right)(s)-T\left(A_{\text {gut }}\right)(s)\right) d s d t \\
& \leq \omega \frac{\gamma}{\beta} \rho\left(A_{\text {gut }}-\widetilde{A}_{\text {gut }}\right) .
\end{aligned}
$$

Note that $T$ is continuous. If $\omega \gamma<\beta$, then $T$ is a monotone decreasing contraction mapping.

\section{Fixed point of monotone contraction mappings}

In this section we discuss an analogue of the Ran and Reurings fixed point theorem [20] in modular function spaces. The key feature in this fixed point theorem is that the Lipschitzian condition on the nonlinear map is only assumed to hold on elements that are comparable in the partial order.

Theorem 3.1 Let $\rho \in \Re$. Let $C \subset L_{\rho}$ be nonempty, $\rho$-closed and $\rho$-bounded. Let $T: C \rightarrow C$ be a monotone increasing contraction mapping. Assume that there exists $f_{0} \in C$ which is comparable to $T\left(f_{0}\right)$, i.e., $f_{0} \leq T\left(f_{0}\right)$ or $T\left(f_{0}\right) \leq f_{0} \rho$-a.e. Then $\left\{T^{n}\left(f_{0}\right)\right\}$ is $\rho$-Cauchy and $\rho$-converges to $\bar{f}$ a fixed point of T. Moreover, if $f \in C$ is comparable to $\bar{f}$, then $\left\{T^{n}(f)\right\}$ $\rho$-converges to $\bar{f}$.

Proof Since $T$ is a monotone increasing contraction, there exists $\alpha<1$ such that

$$
\rho(T(f)-T(g)) \leq \alpha \rho(f-g), \quad \text { for all } f, g \in C, f \leq g \rho \text {-a.e. }
$$


Let $f_{0} \in C$ such that $f_{0}$ and $T\left(f_{0}\right)$ are comparable. Assume that $f_{0} \leq T\left(f_{0}\right) \rho$-a.e. Since $T$ is monotone increasing, we get $T^{n}\left(f_{0}\right) \leq T^{n+k}\left(f_{0}\right)$, for any $n \geq 0$ and $k \geq 1$, which implies

$$
\begin{aligned}
\rho\left(T^{n+k}\left(f_{0}\right)-T^{n}\left(f_{0}\right)\right) & \leq \alpha \rho\left(T^{n+k-1}\left(f_{0}\right)-T^{n-1}\left(f_{0}\right)\right) \\
& \leq \alpha^{n} \rho\left(T^{k}\left(f_{0}\right)-f_{0}\right) \\
& \leq \alpha^{n} \delta_{\rho}(C) \rightarrow 0
\end{aligned}
$$

where $\delta_{\rho}(C)=\sup \{\rho(f-g): f, g \in C\}<\infty$, because $C$ is $\rho$-bounded. Since $\alpha<1$ and $\delta_{\rho}(C)<\infty$, we conclude that $\left\{T^{n}\left(f_{0}\right)\right\}$ is $\rho$-Cauchy. The $\rho$-completeness of $L_{\rho}$ implies the existence of $\bar{f} \in L_{\rho}$ such that $\lim _{n \rightarrow \infty} \rho\left(T^{n}\left(f_{0}\right)-\bar{f}\right)=0$. Because $C$ is $\rho$-closed, we get $\bar{f} \in C$. Since $\left\{T^{n}\left(f_{0}\right)\right\}$ is monotone increasing, the assumptions of Theorem 2.3 are satisfied and will imply that $T^{n}\left(f_{0}\right) \leq \bar{f} \rho$-a.e., for any $n \geq 1$. In particular, we have

$$
\rho\left(T^{n}\left(f_{0}\right)-T(\bar{f})\right) \leq \alpha \rho\left(T^{n-1}\left(f_{0}\right)-\bar{f}\right) .
$$

Since

$$
\begin{aligned}
\rho\left(\frac{\bar{f}-T(\bar{f})}{2}\right) & \leq \rho\left(\bar{f}-T^{n}\left(f_{0}\right)\right)+\rho\left(T^{n}\left(f_{0}\right)-T(\bar{f})\right) \\
& \leq \rho\left(\bar{f}-T^{n}\left(f_{0}\right)\right)+\alpha \rho\left(T^{n-1}\left(f_{0}\right)-\bar{f}\right) \rightarrow 0, \quad \text { as } n \rightarrow \infty,
\end{aligned}
$$

$T(\bar{f})=\bar{f}$, which means that $\bar{f}$ is a fixed point of $T$. Finally let $f \in C$ such that $f$ and $\bar{f}$ are comparable. Since $T$ is monotone, $T^{n}(f)$ and $T^{n}(\bar{f})=\bar{f}$ are comparable. Hence

$$
\rho\left(T^{n}(f)-\bar{f}\right) \leq \alpha^{n} \rho(f-\bar{f}) \leq \alpha^{n} \delta_{\rho}(C), \quad \text { for any } n \geq 1 .
$$

Since $\alpha<1$, we conclude that $\left\{T^{n}(f)\right\} \rho$-converges to $\bar{f}$.

The missing information in Theorem 3.1 is the uniqueness of the fixed point. In fact we do have a partial positive answer to this question. Indeed if $\bar{h}$ and $\bar{g}$ are two fixed points of $T$ such that $\bar{h} \leq \bar{g} \rho$-a.e., then we must have $\bar{h}=\bar{g}$. In general $T$ may have more than one fixed point.

Remark 3.1 The conclusion of Theorem 3.1 does not extend easily to the case of monotone decreasing contractions. Note that the mapping $T$ in Example 2.1 is monotone decreasing. But if we assume that there exists $f_{0} \in C$ such that $f_{0} \leq T^{2}\left(f_{0}\right) \leq T\left(f_{0}\right)$ or $T\left(f_{0}\right) \leq T^{2}\left(f_{0}\right) \leq f_{0} \rho$-a.e., then $\left\{T^{n}\left(f_{0}\right)\right\}$ is $\rho$-Cauchy and $\rho$-converges to $\bar{f}$, a fixed point of $T$. Moreover, if $f \in C$ is comparable to $\bar{f}$, then $\left\{T^{n}(f)\right\} \rho$-converges to $\bar{f}$.

Next we discuss the case of monotone pointwise Lipschitzian mappings and extend the recent results of [2].

Theorem 3.2 Let us assume that $\rho \in \Re$. Let $C \subset L_{\rho}$ be nonempty, $\rho$-closed and $\rho$ bounded. Let $T: C \rightarrow C$ be a pointwise monotone increasing contraction or asymptotic pointwise monotone increasing contraction. Then any two comparable fixed points of $T$ are equal. Moreover, if $f_{0}$ is a fixed point of $T$, then the orbit $\left\{T^{n}(f)\right\} \rho$-converges to $f_{0}$, for any $f \in C$ which is comparable to $f_{0}$. 
Proof Since every pointwise monotone increasing contraction is an asymptotic pointwise monotone increasing contraction, we can assume that $T$ is an asymptotic pointwise monotone increasing contraction, i.e.

$$
\rho\left(T^{n}(f)-T^{n}(g)\right) \leq k_{f}^{n} \rho(f-g)
$$

for any $f \in C$ and $n \geq 1$, and any $g \in C$ comparable to $f$, where $\left\{k_{f}^{n}\right\}$ is such that $\limsup _{n \rightarrow \infty} k_{f}^{n}=k_{f}<1$. Let $f_{1}, f_{2} \in C$ be two fixed points of $T$ such that $f_{1} \leq f_{2} \rho$-a.e. Then we have

$$
\rho\left(f_{1}-f_{2}\right)=\rho\left(T^{n}\left(f_{1}\right)-T^{n}\left(f_{2}\right)\right) \leq k_{f_{1}}^{n} \rho\left(f_{1}-f_{2}\right)
$$

for any $n \geq 1$. If we let $n \rightarrow \infty$, we will get

$$
\rho\left(f_{1}-f_{2}\right) \leq k_{f_{1}} \rho\left(f_{1}-f_{2}\right)
$$

Since $k_{f_{1}}<1$ and $C$ is $\rho$-bounded, we conclude that $\rho\left(f_{1}-f_{2}\right)=0$, i.e. $f_{1}=f_{2}$. This proves the first part of Theorem 3.2. To prove the convergence, assume that $f_{0}$ is a fixed point of $T$. Fix an arbitrary $f \in C$ such that $f \leq f_{0}$ or $f_{0} \leq f \rho$-a.e. Let us prove that $\left\{T^{n}(f)\right\} \rho$-converges to $f_{0}$. Indeed since $T$ is monotone increasing, then $T^{n}(f)$ and $T^{h}\left(f_{0}\right)=f_{0}$ are comparable for any $n, h \geq 1$. Hence

$$
\rho\left(T^{n+m}(f)-f_{0}\right)=\rho\left(T^{n+m}(f)-T^{n}\left(f_{0}\right)\right) \leq k_{f_{0}}^{n} \rho\left(T^{m}(f)-f_{0}\right)
$$

for any $n, m \geq 1$. Hence

$$
\limsup _{m \rightarrow \infty} \rho\left(T^{n+m}(f)-f_{0}\right) \leq \limsup _{m \rightarrow \infty} k_{f_{0}}^{n} \rho\left(T^{m}(f)-f_{0}\right) .
$$

Since $\lim \sup _{m \rightarrow \infty} \rho\left(T^{n+m}(f)-f_{0}\right)=\lim \sup _{m \rightarrow \infty} \rho\left(T^{m}(f)-f_{0}\right)$, we get

$$
\limsup _{m \rightarrow \infty} \rho\left(T^{m}(f)-f_{0}\right) \leq k_{f_{0}}^{n} \limsup _{m \rightarrow \infty} \rho\left(T^{m}(f)-f_{0}\right)
$$

for any $n \geq 1$. If we let $n \rightarrow \infty$, we obtain

$$
\limsup _{m \rightarrow \infty} \rho\left(T^{m}(f)-f_{0}\right) \leq k_{f_{0}} \limsup _{m \rightarrow \infty} \rho\left(T^{m}(f)-f_{0}\right)
$$

Since $k_{f_{0}}<1$, we get $\limsup _{n \rightarrow \infty} \rho\left(T^{n}(f)-f_{0}\right)=0$, which implies the desired conclusion $\lim _{n \rightarrow \infty} \rho\left(T^{n}(f)-f_{0}\right)=0$.

Theorem 3.2 does not discuss the existence part of the fixed point. Next we investigate this problem. We will start our discussion with the existence of fixed point results in the case of uniformly continuous function modulars.

Definition 3.1 Let $\rho \in \Re$. We will say that the function modular $\rho$ is uniformly continuous if for every $\varepsilon>0$ and $L>0$ there exists $\delta>0$ such that

$$
|\rho(g)-\rho(h+g)| \leq \varepsilon, \quad \text { if } \rho(h) \leq \delta \text { and } \rho(g) \leq L, f, g \in L_{\rho}
$$


Let us mention that uniform continuity holds for a large class of function modulars. For instance, it can be proved that in Orlicz spaces over a finite atomless measure [25] or in sequence Orlicz spaces [26] the uniform continuity of the Orlicz modular is equivalent to the $\Delta_{2}$ property.

Lemma 3.1 [2] Let $\rho \in \Re$ be uniformly continuous. Let $K \subset L_{\rho}$ be nonempty, convex, $\rho$ closed, and $\rho$-bounded. Then any $\rho$-type $\tau: K \rightarrow[0, \infty)$ is $\rho$-lower semicontinuous in $K$, where

$$
\tau(g)=\limsup _{n \rightarrow \infty} \rho\left(f_{n}-g\right), \quad \text { for all } g \in K,
$$

where $\left\{f_{n}\right\} \in K$.

Before we state our next result, we need the following definition of a property that plays in the theory of modular function spaces a role similar to the reflexivity in Banach spaces (see e.g. [11]).

Definition 3.2 [11] We say that $L_{\rho}$ has property $(\mathrm{R})$ if and only if every decreasing sequence $\left\{C_{n}\right\}$ of nonempty, $\rho$-bounded, $\rho$-closed, and convex subsets of $L_{\rho}$ has a nonempty intersection.

Theorem 3.3 Assume that $\rho \in \Re$ is uniformly continuous and has property $(R)$. Assume that $\rho$ satisfies the $\Delta_{2}$-property. Let $K \subset L_{\rho}$ be nonempty, convex, $\rho$-closed, and $\rho$-bounded. Let $T: K \rightarrow K$ be a pointwise monotone increasing contraction or asymptotic pointwise monotone increasing contraction. Assume there exists $f \in K$ such that $f$ and $T(f)$ are comparable. Then $T$ has a fixed point $f_{0} \in K$.

Proof Since every pointwise monotone increasing contraction is an asymptotic pointwise monotone increasing contraction, we can assume that $T$ is an asymptotic pointwise monotone increasing contraction. In view of Theorem 3.2, it is enough to show that $T$ has a fixed point. Let us assume that $f \leq T(f) \rho$-a.e. The case when $T(f) \leq f$ is done in a similar way and will be omitted. Set

$$
C=\{g \in K ; f \leq g \rho \text {-a.e. }\} \text {. }
$$

Since $\rho$ satisfies the $\Delta_{2}$-property, $C$ is nonempty, $\rho$-closed, and convex. It is clear that $T(C) \subset C$ since $T$ is monotone increasing. Define the function $\tau: C \rightarrow[0,+\infty)$ by

$$
\tau(g)=\limsup _{n \rightarrow \infty} \rho\left(T^{n}(f)-g\right) .
$$

Note that $\tau(g) \leq \delta_{\rho}(K)$, for any $g \in C$. Lemma 3.1 implies that $\tau$ is $\rho$-lower semicontinuous in $C$. Since $\rho$ has property (R), there exists $f_{0} \in C$ such that

$$
\tau\left(f_{0}\right)=\inf \{\tau(g): g \in C\}
$$

Let us prove that $\tau\left(f_{0}\right)=0$. Indeed, since $f \leq f_{0} \rho$-a.e., for any $n, m \geq 1$ we have

$$
\rho\left(T^{n+m}(f)-T^{m}\left(f_{0}\right)\right) \leq k_{f_{0}}^{m} \rho\left(T^{n}(f)-f_{0}\right) .
$$


If we let $n$ go to infinity, we get $\tau\left(T^{m}\left(f_{0}\right)\right) \leq k_{f_{0}}^{m} \tau\left(f_{0}\right)$, which implies

$$
\tau\left(f_{0}\right)=\inf \{\tau(g): g \in K\} \leq \tau\left(T^{m}\left(f_{0}\right)\right) \leq k_{f_{0}}^{m} \tau\left(f_{0}\right) .
$$

Passing with $m$ to infinity, we get $\tau\left(f_{0}\right) \leq k_{f_{0}} \tau\left(f_{0}\right)$, which forces $\tau\left(f_{0}\right)=0$, as $k_{f_{0}}<1$. Hence, $\limsup _{n \rightarrow \infty} \rho\left(T^{n}(f)-f_{0}\right)=0$ holds, i.e.

$$
\lim _{n \rightarrow \infty} \rho\left(T^{n}(f)-f_{0}\right)=0 .
$$

The $\rho$-continuity of $T$ implies $f_{0}$ is a fixed point of $T$.

Remark 3.2 As we see in the proof of Theorem 3.3, the lower semicontinuity of $\rho$-types is crucial. In [27], it is proved that if $\rho$ is orthogonally additive, and $\left\{f_{n}\right\} \subset L_{\rho}$ is $\rho$-a.e. convergent to 0 such that there exists $\beta>1$ with

$$
\sup _{n} \rho\left(\beta f_{n}\right)=M<\infty
$$

then for any $g \in E_{\rho}$, we have $\lim _{n \rightarrow \infty}\left(\rho\left(f_{n}+g\right)-\rho\left(f_{n}\right)-\rho(g)\right)=0$, which implies

$$
\liminf _{n \rightarrow \infty} \rho\left(f_{n}+g\right)=\liminf _{n \rightarrow \infty} \rho\left(f_{n}\right)+\rho(g) .
$$

We say that $L_{\rho}$ has the $\rho$-a.e. strong Opial property whenever this conclusion is satisfied. In this case, any $\rho$-type is lower semicontinuous in the sense of convergence $\rho$-a.e. This will help prove that if $L_{\rho}$ has the $\rho$-a.e. strong Opial property, $K \subset E_{\rho}$ be a nonempty, $\rho$-a.e. compact, strongly $\rho$-bounded, and convex subset, then any $T: K \rightarrow K$ which is a pointwise monotone increasing contraction or asymptotic pointwise monotone increasing contraction has a fixed point provided there exists $f \in K$ such that $f$ and $T(f)$ are comparable.

Indeed since every pointwise monotone increasing contraction is an asymptotic pointwise monotone increasing contraction we can assume that $T$ is an asymptotic pointwise monotone increasing contraction. Let us assume that $f \leq T(f) \rho$-a.e. The case when $T(f) \leq f$ is done in a similar way and will be omitted. Set

$$
C=\{g \in K ; f \leq g \rho \text {-a.e. }\} \text {. }
$$

Then $C$ is a nonempty, $\rho$-a.e. compact, strongly $\rho$-bounded, and convex subset of $K$. Since $T$ is monotone increasing, we have $T(C) \subset C$. Define $\tau: C \rightarrow[0,+\infty)$ by

$$
\tau(h)=\limsup _{n \rightarrow \infty} \rho\left(T^{n}(f)-h\right) .
$$

Since $C$ is $\rho$-bounded, we have $\tau(h)<+\infty$, for any $h \in C$. Using the properties of $\rho$-types under these assumptions, there exists $f_{0} \in C$ such that

$$
\tau\left(f_{0}\right)=\inf \{\tau(h) ; h \in C\} .
$$

Using the same argument as in the proof of Theorem 3.3, we will get $\tau\left(f_{0}\right)=0$. Hence, $\lim _{n \rightarrow \infty} \rho\left(T^{n}(f)-f_{0}\right)=0$. The $\rho$-continuity of $T$ forces $f_{0}$ to be a fixed point of $T$. 


\section{Competing interests}

The authors declare that they have no competing interests.

\section{Authors' contributions}

All authors contributed equally to the writing of this paper. All authors read and approved the final manuscript.

\section{Author details}

${ }^{1}$ Department of Mathematics \& Statistics, King Fahd University of Petroleum and Minerals, Dhahran, 31261, Saudi Arabia

${ }^{2}$ Department of Mathematics, College of Sciences, King Saud University, Riyadh, Saudi Arabia. ${ }^{3}$ Department of Mathematical Science, The University of Texas at El Paso, El Paso, TX 79968, USA.

\section{Acknowledgements}

The authors would like to extend their sincere appreciation to the Deanship of Scientific Research at King Saud University for funding this Research group No. RG-1435-079.

\section{Received: 21 September 2014 Accepted: 28 January 2015 Published online: 24 February 2015}

\section{References}

1. Kozlowski, WM: Modular Function Spaces. Series of Monographs and Textbooks in Pure and Applied Mathematics, vol. 122. Dekker, New York (1988)

2. Khamsi, MA, Kozlowski, WK: On asymptotic pointwise contractions in modular function spaces. Nonlinear Anal., Theory Methods Appl. 73, 2957-2967 (2010)

3. Khamsi, MA, Kozlowski, WM, Reich, S: Fixed point theory in modular function spaces. Nonlinear Anal. 14, 935-953 (1990)

4. Browder, FE: Nonexpansive nonlinear operators in a Banach space. Proc. Natl. Acad. Sci. USA 54, 1041-1044 (1965)

5. Goebel, K, Reich, S: Uniform Convexity, Hyperbolic Geometry, and Nonexpansive Mappings. Series of Monographs and Textbooks in Pure and Applied Mathematics, vol. 83. Dekker, New York (1984)

6. Gohde, D: Zum Prinzip der kontraktiven Abbildung. Math. Nachr. 30, 251-258 (1965)

7. Kirk, WA: Fixed Point Theory for Nonexpansive Mappings, I and II. Lecture Notes in Mathematics, vol. 886, pp. 485-505. Springer, Berlin (1981)

8. Baillon, JB: Nonexpansive mappings and hyperconvex spaces. Contemp. Math. 72, 11-19 (1988)

9. Goebel, K, Sekowski, T, Stachura, A: Uniform convexity of the hyperbolic metric and fixed points of holomorphic mappings in the Hilbert ball. Nonlinear Anal. 4, 1011-1021 (1980)

10. Khamsi, MA, Kirk, WA: An Introduction to Metric Spaces and Fixed Point Theory. Wiley, New York (2001)

11. Khamsi, MA, Kozlowski, WM, Shutao, C: Some geometrical properties and fixed point theorems in Orlicz spaces. J. Math. Anal. Appl. 155(2), 393-412 (1991)

12. Khamsi, MA: On asymptotically nonexpansive mappings in hyperconvex metric spaces. Proc. Am. Math. Soc. 132, 365-373 (2004)

13. Kirk, WA: Fixed points of asymptotic contractions. J. Math. Anal. Appl. 277, 645-650 (2003)

14. Kirk, WA: Mappings of generalized contractive type. J. Math. Anal. Appl. 32, 567-572 (1970)

15. Hussain, N, Khamsi, MA: On asymptotic pointwise contractions in metric spaces. Nonlinear Anal. 71(10), 4423-4429 (2009)

16. Kirk, WA: Asymptotic pointwise contractions. In: Plenary Lecture, the 8th International Conference on Fixed Point Theory and Its Applications (Chiang Mai University, Thailand, July 16-22, 2007).

17. Kirk, WA, Xu, HK: Asymptotic pointwise contractions. Nonlinear Anal. 69, 4706-4712 (2008)

18. Banach, S: Sur les opérations dans les ensembles abstraits et leur applications aux équations intégrales. Fundam. Math. 3, 133-181 (1922)

19. Nieto, JJ, Rodriguez-Lopez, R: Contractive mapping theorems in partially ordered sets and applications to ordinary differential equations. Order 22(3), 223-239 (2005)

20. Ran, ACM, Reurings, MCB: A fixed point theorem in partially ordered sets and some applications to matrix equations. Proc. Am. Math. Soc. 132(5), 1435-1443 (2004)

21. Jachymski, J: The contraction principle for mappings on a metric space with a graph. Proc. Am. Math. Soc. 136, 1359-1373 (2007)

22. Kozlowski, WM: Notes on modular function spaces I. Comment. Math. 28, 91-104 (1988)

23. Kozlowski, WM: Notes on modular function spaces II. Comment. Math. 28, 105-120 (1988)

24. Graumlich, JF, Ludden, TM, Conry-Cantilena, C, Cantilena, LRJ, Wang, Y, Levine, M: Pharmacokinetic model of ascorbic acid in healthy male volunteers during depletion and repletion. Pharm. Res. 14, 1133-1139 (1997)

25. Shutao, C: Geometry of Orlicz spaces. Diss. Math. 356 (1996)

26. Kaminska, A: On uniform convexity of Orlicz spaces. Indag. Math. 44(1), 27-36 (1982)

27. Khamsi, MA: A convexity property in modular function spaces. Math. Jpn. 44(2), 269-279 (1996) 